\title{
Hábito de fumar en población escolar urbana
}

\author{
Drs. Nelson A. Varyis, ${ }^{* * *}$ Adriana Carretero D., ${ }^{* *}$ M. T. Viviana Salomón, ${ }^{* *}$
} M. T. Blanca Ramírez.** Sr. Eduardo Courbis**

\begin{abstract}
A aross prevalence stody to define some characteristics of the sntoking habits of 565 urban shidents (28y femalesi ages 1.3 to $2 \mathrm{l}$ is presented. $55.6 \%$ of them was tound to be smoker $i 26.3 \%$ for individuals under 14 years ) and $35.8 \%$ of the sample sinokel at least one cigarrette a day.

The mumber nf cigartettes increased according to age and was higher in males.

Suroking was found to beyin more commonly between 1.3 and 14 years of axt. The reasons to begin were imitation $243.4 \%$ of the smokers $)$ and curiosity $\{34,6 \%\}$.

Females started smoking eurlier in lite but males smoked higher amounts of cigarettes. Toxic effects of the latter were widely known and linked to diflerent diseases. The inplications of these results are discussed and control ineasures are proposed.
\end{abstract}

El hábito de consumir tabaco en forma industrializada ha traido, junto con el auge de la industria respectiva, un aumento en la incidencia de aquellas patologias que afectan preferentemente a los thumadores. Es de interés hacer notar que la relación cigarrillo-enfenmedad está sujeta a la dosis de tabaco consumida, al hecho de aspirar o no el humo del producto, a la edad en que se inicia el hábito y a la duración del mismo. ${ }^{1,2, \hbar}$

De diversos estudios realizados sobre el cigarríllo se puede concluir para los fumadores:

- Una mortalidad un $70 \%$ más elevada que en los no fumadores.

- En madres funnadoras: Hijos que pesan en pronedio $200 \mathrm{~g}$ menos que los de madres no fumadoras; aumento del porcentaje de abortos; aumento de riesgo de muerte fetal in útero y aumento del porcentaje de partos prematuros. ${ }^{3}$

- Aumento de la morbilidad más o menos parejo para ambos sexos, comprobándose una mayor incidencia de enfermedades coronarias, afecciones obstructivas de las vías respiratorias, cáncer de diversos órganos como pulmón, lengua, laringe, esófago, vejiga, etc., a la vez que irritaciones del tracto digestivo y alteraciones estéticas en la pigmentación de dedos y dientes. 1,2

- Alteraciones de algunos exámenes de laboratorio. ${ }^{6}$

Considerando estos antecedentes hemos creído oportuno realizar un estudio descriptivo de

\footnotetext{
"Departamento de Pediatria Facultad de Medicita Occidente L'niversidad de Chile.

* Depurtaneillo de Salud Pública y Medicina Social Faciultad de Medicina Oecidente Universidad de Chile.
}

algunas características de este hábito en población escolar urbana, tales como:

- Frecuencia del hábito y edad de iniciación del misma, influencia del medio ambiente sobre la adı̧uisición de éste, cantidad promedio de cigarrillos consumidos por día, conocimiento que se tiene acerca de los daños que el cigantillo origina en la salud.

El conocimiento de las caracteristicas mencionadas es útil para indicar la oportunidad, orientación y profundidad de acciones educativas destinadas a prevenir e] hábito y está incluido dentro. de las recomendaciones internacionales de O.P.S. sobre el tema. ${ }^{5}$

\section{OBJETIVOS}

Nuestra investigación fijó los siguientes objetivos:

1) Establecer la prevalencia del acto y el habito de fumar en la población en estudio por sexo, edad $y$ curso.

2) En los fumadores: establecer la cantidad diaria de cigarrillos fumados por cada grupo etario $y$ por sexo.

3) Establecer la edad de inicio del acto de fumar para el total de la muestra y para cada sexo.

4) Establecer las razones que, en opinión de los encuestados, inducen a fumar.

5) Obtener la proporción de encuestados que consideran nocivo para la salud el hábito de fumar.

6) En opinión de encuestados, determinar las afecciones más frecuentemente relacionactas con el hábito de fumar. 
7) Establecer la tendencia del consumo de cigarrillos durante la semana.

8) Establecer la prevalencia de fumadores entre los mayores de 18 años en la casa de los estudiantes que fuman y de los que no fuman.

\section{MATERIAL Y METODO}

Mediante encuestas aplicadas en mayo de 1980 a 565 alumnos de 1. " y 4." de Enseñanza Media, se obtuvo datos para determinar las características del hábito de fumar en estos cursos. Los alumnos pertenecían al Liceo A 4 de Niñas (289 encuestadas). Miguel Luis Amunátegui (276 encuestados). Ambos liceos se ubican en el Area Occidente de Santiago.

Se diseño una encuesta apropiada a los fìnes de la investigación, la que fue probada y modificada por los encuestadores; alumnos del tercer año de Obstetricia de la Facuitad de Medicina Occidente - Universidad de Chile.

La aplicución de la encuesta se realizó en un mismo día en cada uno de los liceos para evitar transmisión de información de un encuestado a otro.

Se consuitó sobre cantidad de cigarrillos consumidos y se calculó la cantidad promedio diaria, redondeando esta cifra al entero más cercano. rían los individuos con el hábito ya adquirido.

Se definió como "no fumador" al que no fumaba en absoluto.

Se consideró como "gnupo tamiliar" a las personas que habitaban en la misma casa del encuestado en los ailtimos seis meses.

La encuesta se aplicó con ropa de calle, para no inducir respuestas ante la presencia del delantad blanco.

La infomación recolectada fite traspasada a tarjetas perforadas para su proceso.

Las principales caracteristicas de la población estudiada se presentan en las Tablas 1 y 2 .

Los estudiantes encuestados se ubican entre $\operatorname{los} 13 y \operatorname{los} 20$ años de edad con una mayor proporción entre los 16 y 17 años. Los hombres de la muestra tendieron a ser más jóvenes que las mujeres. En lo referente a distribución por curso, los alumnos se ubicaron en forma hornogénea en los cuatro grupos en estudio $\left(1 .^{\circ}\right.$ y $4 .{ }^{\circ}$ Medio de ambos sexos).

\section{RESULTADOS}

La prevalencia de fumadores entre los encuetados se presenta en la Tabla 3. funtar.

En el total de la muestra un $\mathbf{5 5 . 6 \%}$ admitio

En $4 .{ }^{\circ}$ medio esta cifra subió a $62.6 \%$ llegan-

Tabla 1

Población estudiada por edad y sexo

\begin{tabular}{|c|c|c|c|c|c|c|}
\hline \multirow{2}{*}{ Edad } & \multicolumn{2}{|c|}{ Mujeres } & \multicolumn{2}{|c|}{ Hombres } & \multicolumn{2}{|c|}{ Total } \\
\hline & N." & $\%$ & N." & $\%$ & $\mathbf{N}{ }^{\circ}$ & $\%$ \\
\hline-14 & 28 & 9.7 & 29 & 10.5 & 57 & 10.1 \\
\hline $14-15$ años & 75 & 26.0 & 91 & 33,0 & 166 & 29.4 \\
\hline $16-17$ años & 115 & 39.8 & 112 & 40.6 & 227 & 40.2 \\
\hline $18-19$ años & 69 & 23.9 & 42 & 15.2 & 111 & 19.6 \\
\hline 20 y más & 2 & 0.6 & 2 & 0.7 & 4 & 0.7 \\
\hline Total & 289 & 100.0 & 276 & 100.0 & 565 & 100.0 \\
\hline
\end{tabular}

Se consideró “fumador" al que declaró fumar cualquier cantidad por mínima que ella fiuera. $\mathrm{Ca}$ be comentar que ésta es una definición bastante amplia de esta característica.

Se definió como "fumador habitual" al que fumaba, al menos, un cigarrillo diario. Estos se- do en los varones de ese curso a un máximo de $67.9 \%$.

Las cifras de fumadores son superiores en el sexo masculino en los cursos encuestados.

Es importante destacar que ya en $1 .^{\circ}$ medio hay cifras altas de fumadores (48.6\%). 
Tabla 2

Población estudiada por curso y sexo

\begin{tabular}{|c|c|c|c|c|c|c|}
\hline Curso & \multicolumn{2}{|c|}{ Mujeres } & \multicolumn{2}{|c|}{ Hombres } & \multicolumn{2}{|c|}{ Total } \\
\hline 1." Medio & 142 & 49.1 & 142 & 51.4 & 284 & 50.3 \\
\hline $4 .^{\circ}$ Vedio & 147 & 50.9 & 134 & 48.6 & 281 & 49.7 \\
\hline Total & 289 & 100.0 & 278 & 100.0 & 565 & 100.0 \\
\hline
\end{tabular}

Tabla 3

Prevalencia de fumadores según curso y sexo

\begin{tabular}{|c|c|c|c|c|c|c|c|c|}
\hline & \multirow{2}{*}{\multicolumn{2}{|c|}{ Mujeres }} & \multirow{2}{*}{\multicolumn{2}{|c|}{ Hombres }} & \multicolumn{4}{|c|}{ Total } \\
\hline & & & & & \multicolumn{2}{|c|}{ Si } & \multicolumn{2}{|c|}{ No } \\
\hline & N." & $\%$ & N. ${ }^{\circ}$ & $\%$ & N. ${ }^{0}$ & $\%$ & $\mathrm{~N}^{\circ}$ & $\%$ \\
\hline 1.0 & 64 & 45.1 & 47 & 52.1 & 138 & 48.6 & 146 & 51.4 \\
\hline $4 .^{\circ}$ & 85 & 57.8 & 91 & 67.9 & 176 & 62.6 & 105 & 37.4 \\
\hline Total & 149 & 51.6 & 165 & 59.8 & 314 & 55.6 & 251 & 44.4 \\
\hline
\end{tabular}

La variación del acto de fumar por edad y sexo se presenta en la Tabla 4.

\section{Tabla 4}

Variación del acto de fumar por edad y sexo. Distribución porcentual.

Porcentaje de fumadores

\begin{tabular}{|c|c|c|c|}
\hline \multirow{2}{*}{ Edad } & Femenino & Masculino & Total \\
\hline & $\%$ & $\%$ & $\%$ \\
\hline-14 años & 28.6 & 24.1 & 26.3 \\
\hline 14 - 15 años & 49.3 & 58.2 & 54.2 \\
\hline $16 \cdot 17$ años & 51.3 & 62.5 & 56.8 \\
\hline $18-19$ años & 63.8 & 78.6 & 69.4 \\
\hline 20 y más & 50.0 & 100.0 & 75.0 \\
\hline Total & 51.6 & 59.8 & 5.6 .6 \\
\hline
\end{tabular}

De la Tabla 4 se desprende cuue la frecuencia de tumadores aumenta en forma paralela a la edad. La cifra de hombres menores de 14 años era inferior que la cifra femenina pero en el grupo etario siguiente ( 14 años a 15 años) ya la cantidad de hombres fumadores era superior a la de mujeres.

El descenso que la frecuencia de fimadores parece presentar a los 20 años es sólo un artefacto debido a la escasa población de esta categoría etaria en nuestra muestra (sólo 4 individuos).

Al analizar lós datos se encontró además, que el consumo diario de cigarrillo aumentaba proporcionalmente con la edad hasta los 18 años, en que un $84.8 \%$ de los fumadores fumaba más de un cigartillo al día.

De esta tabla se concluye que:

1) Los grupos etarios más jóvenes tenderían a consumir menos cantidad de cigarrillos.

2) La mayoría de los fumadores consumía 1 a 4 cigarrillos diarios.

3) El hábito tendería a aumentas en frecuenvia y a intensificarse con la edad. 
El mismo fenómeno se obserwo por igual en el total de la muestra y en ambos sexos al observarlos separadamente. Además se pudo observar que en los 565 encuestados; 202 (35.8\%) fumaban más de un cigarritlo diario correspondiendo a lo que hemos definido como fiumadores habituales.

Entre un 15 y $33 \%$ de los encuestados de mayor edad tenian un alto consumo diario de cigarrillos ( 10 a 20 al día).

A objeto de precisar la edad de comienzo del acto de fumar se consultó solure este antecedente. Los resultados se presentan en la Tablit 5 .

Se estableció gue la edad de comienzo mis frecuente fiue entre 13 y' 14 años $(64.8 \%)$.

El sexo masculino iniciaria el habito a edad más temprana que el sexo femenimu.

Lats razones rue inducirian a fumar fueron consultadas a los 314 fimadores. Ellos proporcionaron 318 respuestas que se presentan ayrupadas en la Tabla 6 .

\section{Tabla 5}

Edad de comienzo del acto de fimar por sexo

\begin{tabular}{|c|c|c|c|c|c|c|}
\hline \multirow{2}{*}{ Edad } & \multicolumn{2}{|c|}{ Femenino } & \multicolumn{2}{|c|}{ Masculino } & \multicolumn{2}{|c|}{ Total } \\
\hline & N. ${ }^{\circ}$ & $\%$ & $\mathrm{~N}^{\circ}$ & $\%$ & $\mathrm{~N} .{ }^{\circ}$ & $\%$ \\
\hline 10 y menores & 4 & 1.4 & 14 & 5.1 & 18 & 3.2 \\
\hline $11-12$ & 17 & 5.9 & 25 & 9.1 & 42 & 7.4 \\
\hline $13-14$ & 192 & 66.5 & 174 & 63.0 & 366 & 64.8 \\
\hline $15-16$ & 57 & 19.7 & 59 & 21.4 & 116 & 20.5 \\
\hline $17-18$ & 18 & 6.2 & 4 & 1.4 & 22 & 3.9 \\
\hline $19 y$ mbis & l & 0.3 & - & - & 1 & 0.2 \\
\hline Total & 289 & 100.0 & 276 & 100.0 & 565 & 100.0 \\
\hline
\end{tabular}

\section{Tabla 6}

Razones para comenzar a fumar. Distribución por sexo y curso (318 respuestas de 314 fimadores)

\begin{tabular}{|c|c|c|c|c|c|c|c|c|c|c|}
\hline \multirow{3}{*}{ Motivo } & \multicolumn{4}{|c|}{$1^{13}$} & \multicolumn{4}{|c|}{$4 . "$} & \multirow{2}{*}{\multicolumn{2}{|c|}{ Tot:al }} \\
\hline & \multicolumn{2}{|c|}{ Femenino } & \multicolumn{2}{|c|}{ Masculino } & \multicolumn{2}{|c|}{ Femenino } & \multicolumn{2}{|c|}{ Masculino } & & \\
\hline & N." & $\%$ & N." & $\%$ & $\mathrm{~N} \cdot{ }^{\prime \prime}$ & $\%$ & $N . "$ & $\%$ & $\therefore$. & $\%$ \\
\hline $\begin{array}{l}\text { Imitación } \\
\text { at alguien } \\
\text { de casa }\end{array}$ & 10 & 15.7 & 4 & 5.4 & 8 & 9.5 & 7 & 7.4 & 29 & 9.1 \\
\hline $\begin{array}{l}\text { Imitación } \\
\text { a amigos }\end{array}$ & 29 & 4.5 .4 & 32 & 43.3 & 39 & 45.8 & 41 & $4: 3.1$ & 141 & 44.3 \\
\hline Curiosidad & 22 & 34.3 & 32 & 4.3 .3 & 23 & 27.2 & 33 & 34.7 & 110 & 34.6 \\
\hline Prohibicion & 3 & 4.6 & 3 & 4.0 & 6 & 7.0 & 2 & 2.1 & 14 & 4.4 \\
\hline No sabe & - & - & 1 & 1.3 & 2 & 2.3 & 1 & 1.1 & 4 & 1.3 \\
\hline Otros & - & - & 2 & 2.7 & 7 & 8.2 & 11 & 11.6 & 20) & 6.3 \\
\hline Total & 64 & 100 & 74 & 100 & 85 & 100 & 95 & 100 & 318 & 100 \\
\hline
\end{tabular}


Podemos concluir que, para ambos sexos y cursos, las razones que inducen a fumar son: imitación a amigos y curiosidad.

La variación del consumo en el fin de semana se presenta en la Tabla 7.
De la Tabla $\$$ se deduce que casi la totalidad de la población encuestada reconoció efectos tóxicos al cigarrillo; no habiendo diferencias apreciables en esta opinión entre los cursos más jóvenes o mayores.

\section{Tabla 7}

Variación del consumo de cigarrillos en el fin de semana por curso

\begin{tabular}{|c|c|c|c|c|c|c|}
\hline \multirow{2}{*}{$\begin{array}{l}\text { Variación del } \\
\text { fin de semana }\end{array}$} & \multicolumn{2}{|c|}{$10^{\circ}$} & \multicolumn{2}{|c|}{$4 .^{\circ}$} & \multicolumn{2}{|c|}{ Total } \\
\hline & N. ${ }^{0}$ & $\%$ & N. & $\%$ & N." & $\%$ \\
\hline Más & 44 & 31.9 & 91 & 51.7 & 135 & 43.0 \\
\hline Menos & 16 & 11.6 & 15 & 8.5 & 31 & 9.9 \\
\hline Igual & 28 & 20.3 & 50 & 28.4 & 78 & 24.8 \\
\hline Nada & 50 & 36.2 & 20 & 11.4 & 70 & 22,3 \\
\hline Total & 138 & 100 & 176 & 100 & 314 & 100 \\
\hline
\end{tabular}

Los resultados nos merecen los siguientes comentarios:

En el total: un $43.0 \%$ tiende a aumentar el consurno de cigarrillos el fin de semana, un $24.8 \%$ a mentenerlo y sólo un $9.9 \%$ a disminuirlo.

En el análisis por curso: los cuartos medios conservan la tendencia general; en cambio en los primeros medios un alto porcentaje de fumadores (36.2\%) no consume cigarrillos el fin de semana. Esto indicaria que en estos cursos aún no está establecido el habito en forma definitiva.

En la Tabla 8 se presentan datos sobre el reconocimiento de la toxicidad del cigarrillo en el total de los encuestados.
En la Tabla 9 se presenta las afecciones nás fiecuentemente, mencionadas como relacionadas con el hábito de fumar.

La Tabla 10 presenta el antecedente de tener otro fumador en casa en fumadores y no fumadores.

Llama la atención que los fumadores tienen otro fumador en la casa en un $\mathbf{8 4 . 4 \%}$. Los no fumadores sólo tenían en un $79.2 \%$ el mismo antecedente.

A objeto de obtener una medición aproxímada del peso del medio ambiente familiar en el hábito de fumar se preguntó sobre:

Tabla 8

Reconocimiento de la toxicidad del cigarrillo

\begin{tabular}{|c|c|c|c|c|c|c|}
\hline \multirow{2}{*}{ Toxicidad } & \multicolumn{2}{|c|}{$1 .{ }^{\circ}$ Medio } & \multicolumn{2}{|c|}{$4 .^{\circ}$ Medio } & \multicolumn{2}{|c|}{ Total } \\
\hline & $\mathrm{N},{ }^{0}$ & $\%$ & $\mathrm{~N} .^{\circ}$ & $\%$ & N. ${ }^{\circ}$ & $\%$ \\
\hline Sí & 280 & 98.6 & 270 & 96.1 & 550 & 97.3 \\
\hline No & 2 & 0.7 & 8 & 2.8 & 10 & 1.8 \\
\hline No sabe & 2 & 0.7 & 3 & 1.1 & 5 & 0.9 \\
\hline Total & 284 & 100 & 281 & 100 & 565 & 100 \\
\hline
\end{tabular}


a) Número de mayores de 18 años en la casa del encuestado.

b) Cantidad de fumadores entre ellos.

Los resultados obtenidos fueron los siguientes: Los fumadores cohabitaban con 982 mayores de 18 años. De estos últimos fimmabar un total de 531 lo que equivale a un $54.1 \%$. Los no fumadores cohabitaban con 782 mayores de 18 años. De éstos, fimaba un $48.3 \%$.

\section{Tabla 9}

Principales afecciones relacionadas con el cigarrillo en opinión de los estudiantes. (769 respuestas; 565 encuestados)

\begin{tabular}{|c|c|c|}
\hline Afecciones & $\begin{array}{c}\text { Total } \\
\text { Número }\end{array}$ & $\begin{array}{l}\text { Porcentaje } \\
\text { alurunos }\end{array}$ \\
\hline Daño pulmonar & 243 & $45.0 \%$ \\
\hline $\begin{array}{l}\text { Resto aparato respi- } \\
\text { ratorio }\end{array}$ & 137 & $24.2 \%$ \\
\hline Daño embarazada y feto & 84 & $14.9 \%$ \\
\hline Cánceres & 84 & $14.9 \%$ \\
\hline Cáncer Pulmonar & 70 & $12.4 \%$ \\
\hline No sabe cómo & 60 & $10.6 \%$ \\
\hline Otros & 30 & $5.3 \%$ \\
\hline $\begin{array}{l}\text { Daño sistema nervioso } \\
\text { y salud mental }\end{array}$ & 27 & $4.8 \%$ \\
\hline $\begin{array}{l}\text { Capacidad física y } \\
\text { experiencia de vida }\end{array}$ & 22 & $3.9 \%$ \\
\hline $\begin{array}{l}\text { Lesión aparato cardio- } \\
\text { vascular }\end{array}$ & 12 & $2.1 \%$ \\
\hline
\end{tabular}

2) La frecuencia del acto de furnar aumenta con la edad. En menores de 14 años fuma un $26.3 \%$.

3) Un total de 202 individuos $\mathbf{3 5 . 8 \%}$ de los encuestados) consumía uno o más cigarrillos diarios (fumadores habituales).

4) La cantidad que se consume con mayor frecuencia es 1 a 4 cigarillos diarios $\langle 24.8 \%$ de la muestra y $44.6 \%$ de los furnadores).

5) El acto de fumar se iniciaria más precozmente en el sexo masculino, en una seguna etapa el sexo femenino lo practicaría can mayor intensidad $y$, posteriomente, volvería el predominio masculino.

6) Las razones que impulsan a iniciar el acto de fumar son la imitación (53.4\%) y la curiosidad (34.6\%).

7) Existe un amplio reconocimiento de la toxicidad del cigarrillo lo que no impide que los individuos fumen.

\section{RECOMENDACIONES}

1) Los contenidos educabivos destinados a evitar que se inicie el acto de fumar, (previo a la instalación del hábito) deben ser dados a los alumnos antes de los 10 años de edad.

2) La Educación debe iniciarse en el hogar y debe ser complementada con la que se entrega en la educación fornal.

3) La Educación debe tender a evitar que se produzca el fenóneno de imitación que impulsa a fumar a los escolares.

4) Todos aquellos individuos que pudieran

Tabla 10

Presencia de furnadores en casa de encuestados

\begin{tabular}{|c|c|c|c|c|c|c|}
\hline \multirow{2}{*}{$\begin{array}{l}\text { Presencia de } \\
\text { fimadores en } \\
\text { casa }\end{array}$} & \multicolumn{2}{|c|}{ Fumadores } & \multicolumn{2}{|c|}{ No fumadores } & \multicolumn{2}{|c|}{ Total } \\
\hline & N.o & $\%$ & N. ${ }^{0}$ & $\%$ & N. ${ }^{\circ}$ & $\%$ \\
\hline Sí & 265 & 84.4 & 199 & 79.2 & 464 & 82.1 \\
\hline No & 49 & 15.6 & 52 & 20.8 & 101 & 17.9 \\
\hline Total & 314 & 100 & 251 & 100 & 565 & 100 \\
\hline
\end{tabular}

\section{CONCLUSIONES}

1) Con nuestra definición de fumador un $55.6 \%$ de los encuestados presentaría esta característica ( $59.8 \%$ en el sexo masculino). ser tomados como modelo por el nin̈o durante su desarrollo, deben abstenerse de fumar en su presencia.

5) Dadas las proporciones significativas de escolares que fuman una pequeña cantidad (me- 
nos de 1 cigarrillo diario) se debe reforzar, a este nivel, la entrega de contenidos educativos antes que se produzca el hábito.

6) Ante el amplio conocimiento de la toxicjdad del cigarrillo, y dado que el hílvito se hace presente a pesar de ello, es conveniente insistir en otros aspectos negativos de éste que podrian ser más efectivos en su control; por ejemplo: mal aliento, manchas en dientes y manos.

\section{RESUMEN}

Se presentan los resultados de una encuesta sobre hábito de fumar aplicada a 565 estudiantes (289 mujeres) del Area Occidente de Santiago, entre 13 y 21 años.

$55.6 \%$ fumaba ( $26.3 \%$ en las menores de 14 años) y $35.8 \%$ consumía uno o más cigarrillos diarios. El consumo aumentaba con la edad siendo mayor en hombres. La edad de inicio más común fue 13 a 14 años y las razones más frecuentes fueron imitación ( $53.4 \%$ de los fumadores) y curiosidad $(34.6 \%)$.

Las mujeres empezaban a fumar a menor edad pero los hombres consumían mayor cantidad de cigarrillos. Los efectos tóxicos de los últimos eran ampliamente conocidos y relacionados con diferentes enfermedades.

Se analiza el significado de estos resultados y se proponen medidas de control.

Los autores desean agradecer la colaboración y el aporte de los alumnos de 3er. Año de Obstetricia, Facultad de Medicina Oecidente, que hicieron posible la realización de esta investigación.

\section{REFERENCIAS}

${ }^{l}$ Cansex-uencias del Talraco para la bialud. Intemne de comite de Expertos OMS. Serie de Infonmes Tecneos . N." 5xis, OMIS. Ginebre, 1974.

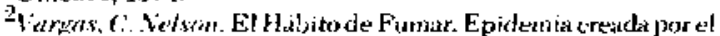
I tombre. Boll. Hospital Sin Juan de Dios. Vol. g7 Y." I. Enerotelyrero, 1940, pise. 24.

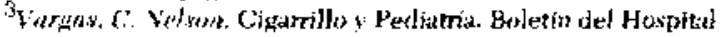
San Juan de Dios. Vol. 25 N. 6 . Voviembre - diciembre 1978, pisg. 354

${ }^{4}$ Highlights of the surgeon keneral s report in smokius and healt. Morbidity and inartality weekly seport center for disease control. Allanta. GA. Jien 1979.

${ }^{5}$ Encilesta sobre las caracteristicas del Hibito de Fumar en Amériez Latina OPS. Pub. Cientílica N. ${ }^{\circ} 337$ Año 1977.

066 Vargas, C.. Velsem; Curbis E. Alteraciones de Exámenes de Lathotatorio en Fumadores Boletín del Hospital Sitn Juan de Dias. Vol. 27 N." 2. Mareo - abril 1980, píy. \$. 